\section{Beyond the lesion: neuroimaging foundations for post-stroke recovery}

Leeanne M Carey*1,2, Rüdiger J Seitz ${ }^{3,4}$, Mark Parsons ${ }^{5,6}$, Christopher Levi ${ }^{5,6}$, Shawna Farquharson ${ }^{7,8}$, Jacques-Donald Tournier ${ }^{7}$, Susan Palmer ${ }^{1}$

\section{\& Alan Connelly ${ }^{7}$}

'Neurorehabilitation \& Recovery, Stroke Division, The Florey Institute of Neuroscience \& Mental Health, University of Melbourne, Melbourne Brain Centre. Austin Campus, 245 Burgundy Street, Heidelberg, Victoria, 3084, Australia

2Department of Occupational Therapy, La Trobe University, Bundoora, Australia

${ }^{3}$ Centre of Neurology \& Neuropsychiatry, LVR-Klinikum Düsseldorf, Germany

${ }^{4}$ Department of Neurology, University Hospital Düsseldorf, Heinrich-Heine-University Düsseldorf; Bergische

Landstrasse 2, 40629 Düsseldorf, Germany

${ }^{5}$ Stroke Program, Centre for Translational Neuroscience \& Mental Health Research, University of Newcastle, Newcastle, Australia

'Hunter Medical Research Institute, Department of Neurology, John Hunter Hospital, Lookout Road, New Lambton, NSW, 2305, Australia

${ }^{7}$ Imaging Division, The Florey Institute of Neuroscience \& Mental Health, Melbourne Brain Centre,

245 Burgundy Street, Heidelberg, 3084, Australia

8Department of Medical Imaging \& Radiation Science, Monash University, Melbourne, Australia

*Author for correspondence: Tel.: +61 390357088 = Fax: +61 390357301 = leeanne.carey@florey.edu.au

A shift is emerging in the way in which we view post-stroke recovery. This shift, supported by evidence from neuroimaging studies, encourages us to look beyond the lesion and to identify viable brain networks with capacity for plasticity. In this article, the authors review current advances in neuroimaging techniques and the new insights that they have contributed. The ability to quantify salvageable tissue, evidence of changes in remote networks, changes of functional and structural connectivity, and alterations in cortical thickness are reviewed in the context of their impact on post-stroke recovery. The value of monitoring spared structural connections and functional connectivity of brain networks within and across hemispheres is highlighted.

\section{Medscape Medscape: Continuing Medical Education Online}

This activity has been planned and implemented in accordance with the Essential Areas and policies of the Accreditation Council for Continuing Medical Education through the joint sponsorship of Medscape, LLC and Future Medicine Ltd. Medscape, LLC is accredited by the ACCME to provide continuing medical education for physicians.

Medscape, LLC designates this journal-based CME activity for a maximum of 1 AMA PRA Category 1 Credit(s) ${ }^{\mathrm{TM}}$. Physicians should claim only the credit commensurate with the extent of their participation in the activity.

All other clinicians completing this activity will be issued a certificate of participation. To participate in this journal CME activity: (1) review the learning objectives and author disclosures; (2) study the education content; (3) take the post-test with a $70 \%$ minimum passing score and complete the evaluation at www.medscape.org/journal/fnl; (4) view/print certificate.

\section{Release date: 28 August 2013; Expiration date: 28 August 2014}

\section{Learning objectives}

Upon completion of this activity, participants should be able to:

- Describe the role of magnetic resonance imaging (MRI) in elucidating structural changes affecting poststroke recovery and management, based on a review

- Identify connectivity of brain regions and networks visualized by neuroimaging techniques in poststroke recovery

- Distinguish functional activation in poststroke recovery as determined by neuroimaging techniques
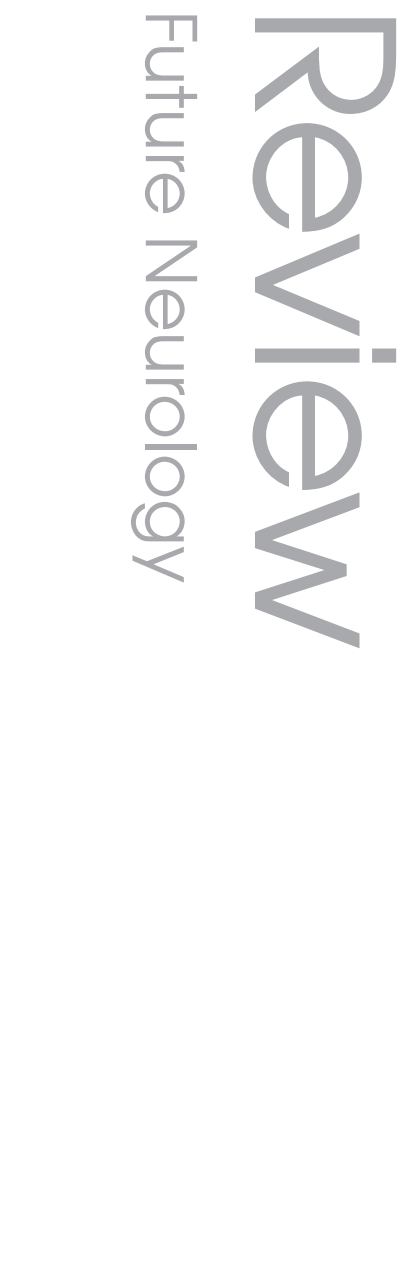

Keywords

- brain networks $=$ cortical thickness $=$ intrinsic functional connectivity $=$ neuroimaging - penumbra = rehabilitation - stroke recovery " white matter tractography

\section{Future Medicine part of}




\section{Financial \& competing interests disclosure}

Editor: Elisa Manzotti, Publisher, Future Science Group. Disclosure: Elisa Manzotti has disclosed no relevant financial relationships.

CME author: Laurie Barclay, MD, is a freelance writer and reviewer, Medscape, LLC. Disclosure: Laurie Barclay, $M D$, has disclosed no relevant financial relationships.

Author \& credentials: Leeanne M Carey, MD, Neurorehabilitation \& Recovery, Stroke Division, The Florey Institute of Neuroscience \& Mental Health, University of Melbourne, Melbourne Brain Centre, Austin Campus, 245 Burgundy Street, Heidelberg, Victoria, 3084, Australia; Department of Occupational Therapy, La Trobe University, Bundoora, Australia. Disclosure: Leeanne M Carey is supported by an Australian Research Council Future Fellowship (\#FT0992299). Leeanne M Carey is grateful to the Victorian Government's Operational Infrastructure Support Program for support.

Rüdiger J Seitz, MD, Centre of Neurology \& Neuropsychiatry, LVR-Klinikum Düsseldorf, Germany; Department of Neurology, University Hospital Düsseldorf, Heinrich-Heine-University Düsseldorf; Bergische Landstrasse 2, 40629 Düsseldorf, Germany. Disclosure: Rüdiger J Seitz has disclosed no relevant financial relationships.

Mark Parsons, MD, Stroke Program, Centre for Translational Neuroscience \& Mental Health Research, University of Newcastle, Newcastle, Australia; Hunter Medical Research Institute, Department of Neurology, John Hunter Hospital, Lookout Road, New Lambton, NSW, 2305, Australia. Disclosure: Mark Parsons has disclosed no relevant financial relationships.

Christopher Levi, MD, Stroke Program, Centre for Translational Neuroscience \& Mental Health Research, University of Newcastle, Newcastle, Australia; Hunter Medical Research Institute, Department of Neurology, John Hunter Hospital, Lookout Road, New Lambton, NSW, 2305, Australia. Disclosure: Christopher Levi has disclosed no relevant financial relationships.

Shawna Farquharson, MD, Imaging Division, The Florey Institute of Neuroscience \& Mental Health, Melbourne Brain Centre, 245 Burgundy Street, Heidelberg, 3084, Australia; Department of Medical Imaging \& Radiation Science, Monash University, Melbourne, Australia. Disclosure: Shawna Farquharson is grateful to the Victorian Government's Operational Infrastructure Support Program for support.

Jacques-Donald Tournier, MD, Imaging Division, The Florey Institute of Neuroscience \& Mental Health, Melbourne Brain Centre, 245 Burgundy Street, Heidelberg, 3084, Australia. Disclosure: Jacques-Donald Tournier is supported by an Australian Research Council Discovery Outstanding Researcher Award (DP130103438). JacquesDonald Tournier is grateful to the Victorian Government's Operational Infrastructure Support Program for support.

Susan Palmer, MD, Neurorehabilitation \& Recovery, Stroke Division, The Florey Institute of Neuroscience \& Mental Health, University of Melbourne, Melbourne Brain Centre, Austin Campus, 245 Burgundy Street, Heidelberg, Victoria, 3084, Australia. Disclosure: Susan Palmer is grateful to the Victorian Government's Operational Infrastructure Support Program for support.

Alan Connelly, MD, Imaging Division, The Florey Institute of Neuroscience \& Mental Health, Melbourne Brain Centre, 245 Burgundy Street, Heidelberg, 3084, Australia. Disclosure: Alan Connelly is grateful to the Victorian Government's Operational Infrastructure Support Program for support.

No writing assistance was utilized in the production of this manuscript.

Stroke is a leading cause of adult disability [1]. One in six people experience a stroke at some time in their life. The global annual incidence is estimated at 15 million [2]. Approximately a third have persistent and significant long-term disability $[2,3]$. Post-stroke recovery varies across patients over time, and according to the definition of recovery used, with $55-75 \%$ of stroke survivors still having functional impairment and reduced quality of life, even months after the infarct [4]. There is strong evidence from animal and human studies for neural plastic changes associated with recovery after stroke $[5,6]$.

Each stroke survivor travels the recovery journey with a different constellation of impairments and capacities. Evidence, both personal and scientific, highlights variable recovery from lesions in similar brain regions [7]. In addition, response to therapy is highly variable. Rehabilitation outcomes are often limited, and some approaches may even lead to deterioration in function by reinforcing imbalances in neural excitability and learned nonuse [8]. Thus, the recovery journey is impacted not only by the initial injury, but also the individual's capacity to respond to specific interventions and therapy.

Stroke impacts extend beyond the lesion site. Focal lesions have important remote effects on the function of distant brain regions that contribute significantly to behavioral deficits observed and to post-stroke recovery [9]. Although damage 
from the initial lesion is focal, remote dysfunction can occur in regions connected to the lesion [10]. For example, changes in remote locations can be observed in the first hour after stroke in animal models [11]. A lesion may also disturb the complex balance of excitatory and inhibitory influences within a network [12]. Knowledge of how brain networks are interrupted is currently limited, but is critical to better understand the nature of the clinical deficit and post-stroke recovery [6]. As the disease process is generally static after the initial insult, subsequent changes in brain function likely represent how the brain responds to injury rather than representing ongoing pathologic processes [10].

A significant challenge is to identify the brain networks and processes mediating functional improvement so that rehabilitation strategies can be aimed at the appropriate targets [5]. The potential for recovery of function and brain adaptation is well established; however, "the rules that govern this are poorly understood" [5]. No single pattern of neuroplastic change is observed during recovery; rather, they are distinctly different in different cerebral regions and seem to depend on deficits caused by the initial lesion, as well as training interventions $[5,13]$.

In this article, the authors review recent advances in our understanding of the neurobiological foundations of post-stroke recovery derived from neuroimaging. Modern neuroimaging techniques provide insight into the anatomy and functioning of the human brain in vivo. Different imaging modalities are suited to investigate different neuroanatomical and functional factors impacting on recovery throughout the recovery process. The authors will focus on four main areas. The first is the characterization of the lesion and the penumbra and how they may impact on the recovery process. Second, the authors will review insights provided by task-related functional neuroimaging techniques and how these may be used to better inform interruption to functional brain regions and the process of recovery. Third, given evidence of remote changes and the importance of brain networks on recovery, the authors review advances in methods to investigate functional and structural brain networks and new insights in our understanding of the impact of brain networks in recovery. Finally, the authors will focus on changes in brain morphology over time and its association with recovery. We conclude by recommending neuroimaging methods that may be used to further our investigations in this area. Future perspectives will speculate on how the field may evolve over the next 5-10 years.

\section{Lesion characterization \& penumbra Lesion characterization}

Ischemic brain infarcts result from cerebral artery occlusions occurring in the brain's gray matter structures, such as the cerebral cortex, the basal ganglia and the thalamus or in the white matter. The immediate changes of ischemia are apparent from perfusion imaging and completely disappear upon reperfusion [14]. The first structural changes of the brain due to cytotoxic cell swelling can be shown with diffusion-weighted MRI within $2 \mathrm{~h}$ after the ischemic event and largely predict the remaining infarct lesion [15-17]. The structural brain lesions undergo a dynamic evolution owing to secondary changes, such as tissue degradation and brain edema formation, and a subsequent lesion shrinkage accompanied by atrophy of the ventricles and the cerebral or cerebellar cortex [18]. After approximately 30 days the infarct lesions are largely stable and clearly visible in T2 and T1 magnetic resonance (MR) images. Table 1 outlines changes observed for different MRI sequences during the evolution of the immediate ischemic changes to the structural infarct lesion. The location of the infarct lesions can be communicated with respect to gross anatomical landmarks or in relation to the stereotaxic system [19]. Owing to the semiquantitative character of MRI, lesion volumes can be assessed for clinical purposes by simple inspection, while in the scientific context, a plethora of different approaches has been advocated [20,21].

The severity of the neurological deficit following a brain infarct depends on the interval until artery recanalization and the location and volume of the infarct lesion [22]. While the neurological deficit typically regresses virtually completely after small cortical infarcts owing to perilesional plasticity $[23,24]$, brain infarcts affecting white matter tracts result in complex and/or

\begin{tabular}{|c|c|c|c|c|c|}
\hline Sequence & $\begin{array}{l}\text { Hyperacute } \\
<3 \mathrm{~h}\end{array}$ & $\begin{array}{l}\text { Acute } \\
3-24 \mathrm{~h}\end{array}$ & $\begin{array}{l}\text { Subacute } \\
1-14 \text { days }\end{array}$ & $\begin{array}{l}\text { Chronic } \\
1-6 \text { months }\end{array}$ & $\begin{array}{l}\text { Residual } \\
>6 \text { months }\end{array}$ \\
\hline PWI & ++ & + & - & - & - \\
\hline DTI/ADC & $+/-$ & ++ & $+/-$ & - & - \\
\hline $\mathrm{T} 2$ & - & $+/-$ & ++ & + & $+/-$ \\
\hline FLAIR & - & $+/-$ & ++ & + & $+/-$ \\
\hline DTI/DWI & - & - & $+/-$ & + & + \\
\hline \multicolumn{6}{|c|}{$\begin{array}{l}\text {-: Changes not evident at this time; +: Changes present and able to be detected at this time; } \\
\text { ++: Changes strongly present at this time; +--: Changes sometimes present/are able to be detected; } \\
\text { ADC: Apparent diffusion coefficient; DTI: Diffusion tensor imaging; DWI: Diffusion-weighted } \\
\text { imaging; FLAIR: Fluid attenuated inversion recovery; PWI: Perfusion-weighted imaging. }\end{array}$} \\
\hline
\end{tabular}


persistent neurological deficits [25,26]. Notably, however, even small cortical infarcts that allow for profound recovery of the acute neurological deficit bear the risk of substantial morbidity similar to large brain infarcts [27].

\section{Penumbra \& early diffusion changes}

Early recovery (within the first few days after stroke) relates to reperfusion and salvage of the ischemic penumbra. The ischemic penumbra is tissue that is acutely hypoperfused to an extent that leads to impairment of brain function and clinical symptoms. Generally this tissue will become irreversibly damaged if adequate perfusion is not restored in a timely fashion. With advanced computed tomography (CT) and MR neuroimaging, there are clinically available tools that can provide insights into volumes and topography of penumbra and infarct core. This information is increasingly being used to select patients for acute reperfusion therapy and may be used to assist prediction of chance of early and late stroke recovery. However, we do not yet have level 1 evidence for the use of advanced neuroimaging in this manner. The initial imaging studies of penumbra focused on PET, but this is not clinically practical. Current widely used methods to image penumbra and infarct core are diffusion/perfusion MRI and perfusion CT. Diffusion MRI images the infarct core and perfusion MRI measures the passage of an intravenous (paramagnetic) contrast through the brain to generate various measures of perfusion. Perfusion CT uses a similar approach to perfusion MR (using iodinated contrast) to generate various measures of perfusion. MRI has the advantage of diffusion MR, which has been shown to be a very accurate measure of infarct core within minutes of onset of ischemia. Perfusion CT is faster and more accessible, but has the disadvantage that thresholds of reduced perfusion must be used to measure both infarct core and penumbra. The use of such thresholds varies considerably across studies (both perfusion MR and CT) and needs standardization before it will fully translate into clinical practice [28].

The degree of early recovery after stroke depends firstly upon the balance between the volume and topography of irreversible injury (infarct core) versus the volume and topography of salvageable tissue (penumbra) [14]. This varies dramatically from patient-to-patient and particularly relates predominantly to the adequacy of collateral flow from leptomeningeal collaterals from unaffected arteries that anastomose with distal branches of the occluded vessel, supplying retrograde blood flow to the ischemic region [29]. Provided there is not extensive infarction before early reperfusion, early stroke recovery relates to the extent of early reperfusion, particularly in how much of the penumbra it successfully restores perfusion to without irreversible injury (infarction) [30]. Smaller volumes of more strategically placed infarction may also contribute to lack of early (and perhaps late) recovery [31]. However, in general, the earlier and more completely reperfusion occurs, the greater the chance of marked early recovery and also an excellent longer-term recovery (Figure 1) [32].

Although generally it is considered that lack of adequate reperfusion equates to poor recovery by leading to conversion of the penumbra to infarction, the role of persistent, less severe, hypoperfusion without infarction, and how it may hinder late recovery - owing to, for example, impairment of plasticity - is poorly understood. Furthermore, whether all reperfused penumbral tissue that does not progress to infarction (evident on structural imaging) is truly healthy and can contribute to normal function, or indeed, can contribute to brain networks in later recovery, has not to our knowledge been studied.

\section{Functional task-related activation \& recovery Task-related functional MRI to identify brain regions \& networks}

Functional neuroimaging techniques, such as functional MRI (fMRI) and functional taskrelated PET, permit interrogation of changes in brain activity at a whole brain level. They enable monitoring of ongoing brain activity in vivo and can be used to identify regions in the brain that are activated in response to experimentally controlled stimulation or other task performance. Activation maps derived may be correlated with performance, compared between groups to identify regions of difference or monitored over time to determine change associated with recovery.

fMRI has become the primary tool to investigate these changes. It is a noninvasive method that allows visualization of patterns of activity in the brain during movement, sensation, cognition and language. The fMRI signal may be acquired using a 'block' or 'event-related' design [33] and is often combined with tracking of participants' responses and reaction times. fMRI measures brain activity by detecting associated changes in blood flow, on the basis that cerebral blood flow and neuronal activation are coupled [34]. The primary form of fMRI uses blood oxygen level-dependent (BOLD) contrast that is based on the hemodynamic response related to energy 
use by brain cells [35]. Data are preprocessed to account for noise in the data prior to the statistical search for task-related activation. Analysis typically includes use of the univariate general linear model model that involves analysis of data at each voxel. Anatomical location can be objectively quantified using coordinate systems, such as that of the Montreal Neurologic Institute (Montreal, Canada), or probabilistic cytoarchitectonic maps [36]. MRI typically has better spatial resolution than electroencephalography and magnetoencephalography; however, temporal resolution of $\mathrm{fMRI}$ is limited. Reliance on the assumption that the BOLD response is stable after stroke has been questioned and may lead to underestimation of brain activity in patients with stroke, highlighting the need for caution in interpretation of data [37].

Task-related fMRI and PET have been used to investigate changes in brain activation associated with recovery and in response to rehabilitation. These changes are often interpreted as evidence of neural plastic change following stroke. Three key patterns of change in brain activation may be described:

- Reorganization: involving activation of existing brain regions or networks that are not typically recruited for the particular task. For example, secondary sensorimotor areas [38], contralesional regions [39] and related networks [40] may be active during a simple motor task that would not normally require activation of these areas, as determined by comparison with healthy controls;

- Remapping: this may involve an expansion or shift in the expected cortical representation for a particular function such as movement. For example, motor recovery has been commonly associated with a shift in the expected activation of the motor cortex to the posterior somatosensory region [41,42]. Activation in the region surrounding the infarct (perilesional activation) has also been described [43];

- Rebalance: there may also be a change in the balance of activity across hemispheres. This may involve changes in the presence and extent of activation in the lesioned and contralesional hemispheres [44].

The growing body of evidence in this field has culminated in a number of systematic reviews and meta-analyses. Most studies of upper limb motor recovery have recruited stroke survivors in the chronic phase [45,46]. Others have investigated change over time $[38,47]$. A quantitative synthesis of findings has been reported using meta-analysis

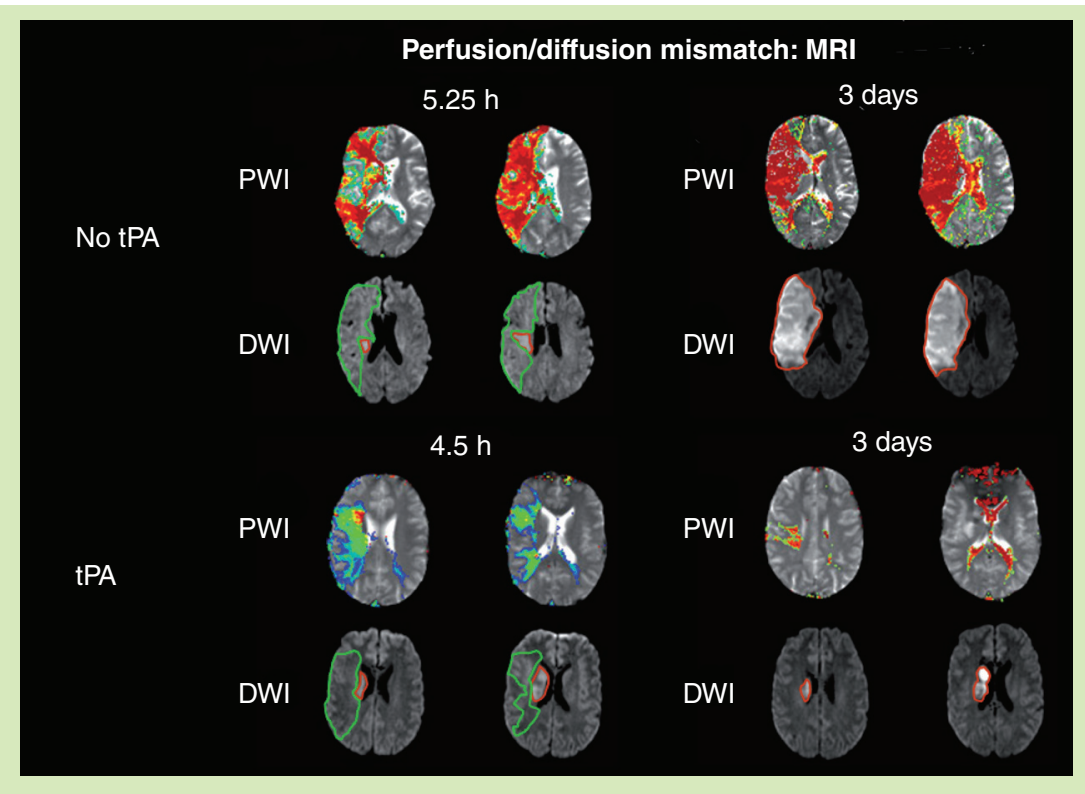

Figure 1. Examples of natural history of the penumbra and how it may be altered by a therapy that augments early reperfusion and salvages penumbra. Top half of the figure shows PWI and DWI acutely and at 3 days in a patient who received no acute reperfusion treatment (natural history example). The PWI lesion (green outline) is much larger than the DWI lesion (red outline). This so-called 'perfusion/diffusion' mismatch represents the ischemic penumbra. On follow-up MRI, a large PWI lesion remains and the DWI lesion has grown substantially, demonstrating that virtually all the penumbral region progressed to infarction, with the consequence being a very poor clinical recovery. This should be compared with the patient images in the lower half of the figure. This patient has a similar acute MRI profile with a large PWI lesion and small DWI lesion (perfusion/diffusion mismatch) representing a large penumbra (green outline). This patient received acute reperfusion therapy with a thrombolytic drug (tPA) and subsequently there was almost complete tissue reperfusion on follow-up PWI, with complete salvage of the penumbra from infarction (the DWI lesion on follow-up did not grow). This translated into excellent early (and long-term) clinical recovery. DWI: Diffusion-weighted imaging; PWI: Perfusion-weighted imaging; tPA: Tissue plasminogen activator.

and the coordinate-based activation likelihood estimation technique [48]. Patients across studies showed greater activation in contralesional primary motor cortex (M1), bilateral ventral premotor cortex (PMC) and supplementary motor area (SMA) relative to healthy subjects. Better motor performance was associated with greater activation likelihood in ipsilesional M1, pre-SMA, contralesional PMC and the cerebellum. Consistent with previous reviews, a good functional outcome was associated with recruitment of the original functional network rather than contralesional activity. A systematic review of serial neuroimaging studies in the first 6 months post-stroke revealed unilateral overactivation of motor and nonmotor areas, a posterior shift in M1 and bilateral recruitment of associated motor and nonmotor areas [41]. Good recovery was associated with return to more normal patterns of activation, while poor recovery was associated with 
persistent, contralesional activation [41]. Recently, the authors have reviewed studies of participants in the first month post-stroke and found increased activation in sensorimotor regions, including the SMA and PMC compared with healthy controls and poor recovers [49]. Recovery of other functions, such as language [50], attention [51] and somatosensation [52,53], have also been linked with reorganization in secondary and contralesional areas and normalization of activation. Differences in patterns of reorganization have been identified following cortical or subcortical lesions; for example, in relation to motor [54] and somatosensory [53] recovery. These findings suggest lesion-specific mechanisms of brain adaption and different rehabilitative strategies to target specific brain regions.

Evidence that specific interventions facilitate neural plasticity and improved motor recovery after stroke in humans is growing $[45,46,55,56]$. To date, 19 studies and two systematic reviews have been identified [49]. Interventions investigated include constraint-induced movement therapy [57-59] and repetitive task-specific training [60]. Other interventions include bilateral arm training [61], mental practice [62] and mirror therapy [63]. Most studies use small numbers and a few have used randomized control methodology. Systematic reviews have identified neural changes in the ipsilesional sensorimotor cortex [46] and ipsilesional and contralesional motor and nonmotor areas [45] in chronic stroke survivors, including the bilateral PMC, the SMA and the cerebellum [49]. Whilst this evidence suggests some common brain regions and networks associated with specific interventions, there are also differences. For example, following bilateral and unilateral arm training [61], suggesting that the therapies operate through different mechanisms. These differences may, at least in part, explain individual differences in ability to benefit from rehabilitation after interruption of different brain networks and highlight potential targets for therapeutic interventions.

\section{Brain networks \& impact on recovery} White matter fiber connectivity \& integrity

The possibility of evaluating the structural characteristics ('integrity') and connectivity of white matter fiber tracts of both those that are within the lesion, as well as those beyond the lesion, which may be affecting function, represents an important step in the prediction and potential recovery of those affected by stroke. Important developments in diffusion-weighted imaging (DWI) analysis methods mean that we now have the ability to visualize a range of white matter pathways supporting eloquent functions in vivo using DWI-based tractography methods.

Diffusion-based tractography exploits the principle that highly structured and oriented tissue structures, as are found in white matter, strongly influence the observed macroscopic diffusion of water molecules (i.e., their random thermal, microscopic and translational motion [64]). The diffusion-weighted signal, being sensitive to this diffusion, displays a dependence on the orientation along which diffusion is measured. The measured DWI signal is modeled at a macroscopic level to provide estimates of fiber orientations within individual voxels. It is these fiber orientation estimates that form the basis of diffusion-based tractography methods, which aim to reconstruct the complex architecture of white matter fiber pathways in 3D [65].

To date, diffusion-based techniques have been used to investigate two main issues: first, in the investigation of white matter fiber connectivity - specifically, the relationship between the lesion and cortical connections [66-69], and second, to quantify the structural integrity of pathways, such as the corticospinal tract (CST) $[66,69,70]$. Common themes that have driven much of the clinical research in this area have been the prediction of outcomes following stroke [71,72] and the measurement of improvements in stroke outcomes by the application of appropriate targeting of therapeutic strategies [55,73]. There is now increasing imaging evidence to suggest that motor outcome post-stroke is related to the extent of the CST damage and the integrity of affected white matter pathways and not limited to infarct size or volume [20,67,71,74-76]. Initial studies demonstrate that diffusion anisotropy in regions along the CST distal to the infarct may be a measure that could be used to predict the amount of motor improvement in response to training $[71,73]$. However, although there have been many promising findings to date, the majority of diffusion studies are based on a fundamentally flawed model to estimate white matter fiber orientations from DWI data, namely the diffusion tensor imaging (DTI) model [77].

The main limitation of the DTI model for the purposes of tractography is that multiple fiber populations can be observed in over $90 \%$ of imaging voxels [78], and DTI can only resolve a single fiber orientation within individual voxels. Farquharson et al. have recently demonstrated the severe limitations of using DTI-based tractography techniques for the purposes of reconstructing tracts projecting to and from the sensorimotor 
cortex [79]. In this comprehensive study, the CST tractography results of DTI-based tractography methods are directly compared with those generated using more a more advanced tractography technique (constrained spherical deconvolution [80]), showing that DTI-based techniques result in systematically unreliable and clinically misleading information [79]. These issues are well known within the technical DWI community [65,78], and are entirely consistent with many of the tractography studies presented to date. This may, therefore, provide an explanation for the apparent inconsistencies currently in the stroke tractography literature $[66,67,69-71]$. Over the past decade, more advanced models have been developed to specifically address the limitations of the DTI model [65]. These developments provide a more robust characterization of the fiber orientation estimates within individual voxels. The advantages of utilizing these more advanced models for clinical research applications are only now being realized.

Current research demonstrates that it is now possible to generate biologically reliable tractography information (Figure 2) to assess structural connections of targeted pathways [79,81] and also to perform quantitative analyses using novel voxel-based comparisons that utilize measures derived from these improved models [82-84]. These exciting developments mean that it may now be possible to extend DWI techniques beyond providing an early marker for stroke toward the provision of accurate information about the structural integrity of white matter fiber tracts, within and beyond the lesion. This may prove to be invaluable in the prediction and potential recovery of those affected by stroke.

\section{Functional connectivity of brain networks}

One of the foundation principles of neuroscience is that neurons do not function in isolation. They are organized into neural networks that act together. These networks communicate not only during a task, but also at rest. The evolution of neuroimaging to permit the examination of network functions is an exciting development to better understand the dynamic process of recovery after stroke. In particular, it allows investigation of the communication across hemispheres and within and between distributed networks that interact and may have a role in recovery. In this way it permits a more comprehensive and dynamic understanding of the effects of stroke and their impact on recovery.

One method to investigate functional brain networks that has evolved over the past decade and is growing in popularity is intrinsic functional connectivity (FC) MRI [85]. The method, also referred to as 'resting-state' FC, can be used to efficiently investigate the integrity of multiple networks. It measures the temporal coherence of low-frequency fluctuations $(<0.1 \mathrm{~Hz})$ of the BOLD signal between spatially remote brain regions. Spontaneous fluctuations in the BOLD signal are acquired during an extended, nondemanding, uncontrolled 'resting' brain state. This neurophysiological index of FC is linked to synchronization of slow fluctuations in the underlying neuronal signals [86]. An important advantage of the method is that the data can be acquired over 5-10 min even when the subject is unable to perform a particular task (as after stroke). It can be used to image subjects with a broad range of impairments and a single scan permits study of multiple networks simultaneously [10]. Networks can be reliably detected using this method, although connectivity data may be influenced by the MRI experience before and during acquisition [87,88]. Resting-state FC has a modest to good test-retest reliability, which is important for longitudinal study designs [89,90]. A potential limitation is that the behavioral measure is obtained in a different session.

Data are analyzed for coherence across the whole brain and/or in relation to particular regions of interest. The analysis may be conducted in a data driven fashion across the whole brain using methods such as independent component analysis [91] or graph theoretical models [92]. This approach has the advantage of identifying

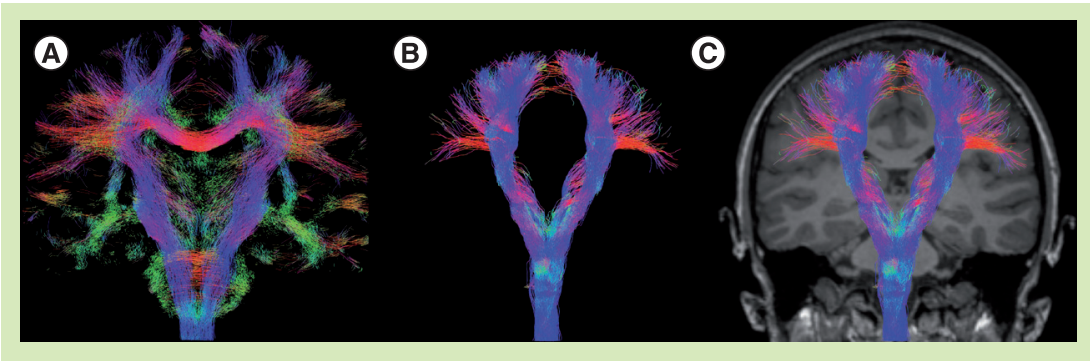

Figure 2. An example of tractography results that can be obtained using advanced methods and relatively modest clinical scans $\left(b=3000 \mathrm{~s} / \mathrm{mm}^{2}\right.$, 60 diffusion-weighted directions, $2.5 \mathrm{~mm}$ voxel size, $<10 \mathrm{~min}$ scan time on Siemens 3T Trio ${ }^{\text {TM }}$ system; Erlangen, Germany). (A) A 2-mm-thick slice through 100,000 whole-brain tracks generated by initiating tracking from random locations throughout the brain. (B) Delineation of the corticospinal tracts generated by initiating tracking from the sensorimotor region, and selecting only those tracks that reach the pons; note the presence of the lateral projections that are often absent from diffusion tensor imaging-derived results. (C) The same results overlaid on a coregistered high-resolution magnetization prepared rapid gradient echo image for anatomical reference. All results were derived using constrained spherical deconvolution [80] for fiber orientation estimation and probabilistic streamlines for track propagation [84]. 
multiple networks and their relationships but requires meaningful interpretation guided by theory. Alternatively, a hypothesis-driven 'seedbased' approach asks the question 'what areas across the whole brain show synchrony with this region of interest?' [93]. The strength and location of connectivity in individuals and in groups is then quantified from the FC maps. In comparison, graph theory analyses quantify the topology of a network. Features of the network such as nodes (corresponding to brain regions) and connected edges (inter-regional interactions) are defined. Measures of the properties or efficiency of the network such as 'centrality' and 'betweeness' may also be obtained [92,94]. Within a network certain brain regions that have more widespread connections and mediate functional integration between regions have been referred to as 'hubs' [92]. Effective connectivity between networks may be assessed using methods such as dynamic causal modeling [95] and structural equation modeling [96]. These mathematical models are usually applied to task-based imaging data using a hypothesis-testing approach to estimate interactions in a predefined network of brain regions. The analysis shows which nodes in a network are driving other nodes, thus providing important information about the direction of information flow.

Consistent resting-state networks, with sharp transitions in correlation patterns, are reliably detected in individual and group data [97,98]. They show high retest reliability and can be reliably pooled across centers [91]. Various networks have been characterized, including visual, auditory, somatosensory and motor, as well as a number of associative 'control' networks, such as the default, dorsal attention, fronto-parietal and ventral attention [93,99]. Resting-state activity is associated with, predicts, and is modified by behavioral performance and by learning over time [100-102]. Strong associations between functional and structural connectivity networks have been revealed in monkeys [103] and humans [104].

Local and global changes in FC have been described after stroke $[10,105,106]$. Change in the strength of intrinsic functional connections between hemispheres and between nodes within a network have been described in a few studies; most showing an association with behavioral deficits [9,12,107-114]. The most consistent finding is of changes in interhemispheric intrinsic FC between homotopic areas, such as ipsilesional and contralesional M1 [9,109,111]. Changes in frontal and parietal cortices, bilateral thalamus, cerebellum and occipital cortex have also been reported [111].
Reduced interhemispheric connectivity was also found between the attention-related areas in stroke patients with neglect $[9,107,115]$, between the language areas in stroke patients with aphasia [116] and between somatosensory areas with sensory loss [117]. Evidence suggests that disruption of interhemispheric connectivity in the somatomotor network and the dorsal attention network is more strongly associated with behavioral impairment in those domains than is intrahemispheric connectivity [9]. Reduced interhemispheric FC coincides with degeneration of transcallosal fibers between M1 cortices of rats [118] and may be influenced by the degree of structural damage to the CST [10]. Remote effects appear to remain within the bounds of the existing network connections. For example, double dissociation of two cognitive control networks in patients with focal brain lesions found a correlated decrease in intrinsic FC within the lesioned network while sparing the nonlesioned network [115]. Differences in resting-state networks are also consistent with altered task-related activation underlying behavioral deficits [107].

Longitudinal changes have also been reported. Decreased interhemispheric FC of the ipsilesional sensorimotor cortex has been reported early after stroke, with return to more normal levels during the recovery process $[9,109,111,114]$. Further FC of ipsilesional M1 with contralesional thalamus, SMA and middle frontal gyrus at onset was positively correlated with motor recovery at 6 months [111]. Wang et al. scanned subcortical patients across five timepoints over a single year [109]. Using a FC matrix and graph theoretical analysis, findings revealed increased regional centrality in ipsilesional M1 and contralesional cerebellum and decreased centrality in ipsilesional cerebellum; in association with recovery. A shift towards a more random configuration of the motor execution network was reported during the process of recovery and correlated with motor recovery [109].

Changes in effective connectivity associated with task-related activation over time have also been investigated in a few studies [40,119-124]. For example, Grefkes et al. report reduced interhemispheric interactions between M1 cortices, and between ipsilesional SMA and contralesional M1 in association with hand motor disability after subcortical stroke [119]. Reduced connectivity has also been demonstrated between the SMA and the ipsilesional PMC in both imagined and executed tasks [40]. Decreased effective resting-state connectively between fronto-parietal regions and M1 and SMA have 
been reported compared with healthy controls [124], highlighting the potential importance of top-down fronto-parietal attention regions. A pictorial summary of changes in intrinsic FC and effective connectivity has recently been provided in relation to motor recovery [106]. Nodes represented include M1, SMA, PMC, prefrontal cortex, superior parietal cortex, cerebellum and thalamus bilaterally. While alterations in interhemispheric connections are highlighted with intrinsic FC, effective connectivity findings highlight reduced intrahemispheric interactions in the ipsilesional hemisphere [106]. These include reduced excitatory influence from fronto-parietal areas especially PMC on ipsilesional M1. Disturbed interhemispheric effective connectivity between M1 regions appears to depend on time since stroke and motor impairment. Changes in resting FC has also been revealed with magnetoencephalography [125], with motor recovery predicted by increased connectivity in ipsilesional SI, SMA and cerebellum and reduced connectivity in contralesional motor regions.

Intrinsic FC outcomes have been investigated in relation to a few interventions in stroke survivors: robot-aided motor rehabilitation [126]; brain-computer interface with motor imagery [127]; noradrenergic stimulation [123]; and an upper extremity accelerated skill acquisition program [108]. A greater reduction in intrinsic FC between ipsilesional and contralesional M1 was associated with greater gains in motor function after 12 weeks of robot-aided motor rehabilitation [126]. In relation to motor imagery, FC changes involving SMA, ipsilesional and contralesional M1, visual areas and cerebellum, predicted individual gain in motor function [127]. Following a skill acquisition program, improvement was associated with increased influence on bilateral PMC and ipsilesional M1 [108]. Intervention studies using effective connectivity support a crucial role of premotor-M1 connectivity [121]. Together these findings highlight the important role of brain networks in recovery, even at rest. Further investigation is required to determine the extent to which patterns of FC predict behavioral deficits, the extent to which they are robust across sessions, whether they correlate with changes in behavior over time and how they may change with rehabilitation [10].

\section{Morphological changes over time Gray matter changes over time}

Changes in brain morphology over time in both the perilesional region and in remote regions may also impact on recovery. Cortical thickness mapping
[128] and voxel-based morphology (VBM) [129] are two methods that have been developed and are being refined for this purpose. These advanced imaging methods have the value that they may detect subtle changes over time and/or in relation to morphology of control groups. However, caution needs to be executed in their application to stroke given the local changes in morphology introduced by the lesion. In particular, factors such as presence of edema, damage to cortical structures owing to the lesion, and the impact of this damage on measurements within the regions and across regions have not been resolved.

Voxel-based and surface-based methods of analysis have been employed. VBM is an analysis technique that involves a voxel-wise comparison of the local concentration of gray matter. VBM registers every brain to a template. The image volume is then compared across the entire brain at every voxel [129]. Comparisons may be made between groups of subjects or over time. Cortical thickness mapping estimates the thickness of the human cerebral cortex [128]. The focus is on the surface of the cortex, although the method may also be used to measure other structures, such as the hippocampus and thalamus. The MRI data acquisition is typically performed on $1.5 \mathrm{~T}$ and $3 \mathrm{~T}$ systems using a T1-weighted 3D sequence, such as magnetization prepared rapid acquisition with gradient echoes with an isotropic spatial resolution of $1 \mathrm{~mm}$. Brain volume data are segmented into white matter, gray matter and cerebrospinal fluid. The cortical thickness is then calculated as the distance between the white matter-gray matter surface and the gray matter-cerebrospinal fluid surface. Different analysis approaches have been developed and compared [128]. Methods have been refined to maximise accuracy and reproducibility, achieve automated analysis and address partial volume effects [130]. Accurate cortical thickness estimation is important for the study of many neurodegenerative diseases, but is also promising for capturing the subtle brain tissue shrinkage in perilesional and remote locations after stroke.

Changes in brain morphology over time may be associated with neurological performance and/or deterioration in performance over time. The first report showing such a relation using VBM was by Kraemer et al. [18]. Atrophy in ipsilesional thalamus and enlargement of lateral ventricles was found in ten stroke patients from 1 month to 2.5 years post-stroke. There is some evidence that areas such as the hippocampus and thalamus show greater atrophy after stroke, and this may be associated with cognitive decline at 
3-6 months post-stroke [131]. In comparison, hippocampal volume was investigated in a sample of 90 stroke patients cross-sectionally, but did not find a difference at 3-6 months post-stroke compared with controls [132]. The rate of brain atrophy from 3 to 12 months post-stroke is reported to be more than twice that of age-matched controls [133]. More recently, cortical thickness changes have been described in an exploratory analysis of ten stroke patients from $<3 \mathrm{~h}$ to 3 months poststroke [134]. Percentage change scores in cortical thickness were reported in contralesional paracentral superior frontal and insular regions, as well as a significant reduction in thalamic volume ipsilesionally. Most studies used a priori regions of interest. Patients with lesions in these regions are excluded from analyses of these brain regions.

Changes in cortical thickness of brain regions have also been associated with structural plasticity and remodeling. Changes have been observed in sensorimotor cortical areas of animals [135] and humans in response to manipulations of specific experience; for example, in London taxi drivers [136] and with learning [137]. Schaechter et al. reported the first evidence of change in cortical thickness in the postcentral gyrus in stroke patients [138]. The change, referred to as structural plasticity, was colocalized with areas of functional plasticity associated with motor recovery in the same individuals. Changes in brain volume have also been investigated in response to specific interventions. Gauthier et al. found marked changes in bilateral sensory and motor areas and hippocampi in the group receiving constraint-induced movement therapy $(\mathrm{n}=16)$ compared with the comparison therapy group $(n=20)$ [139]. Furthermore, the magnitude of gray matter increase, measured using VBM, correlated with the amount of improvement in real-world arm use. Changes occurred after 2 weeks of therapy highlighting the rapid time course of change. Whether the changes are a cause or effect of the behavioral changes is unclear, as are the mechnisms underlying these changes. While evidence of changes after stroke using VBM and cortical thickness estimates are still relatively rare and issues need to be addressed in relation to analyses in the presence of a lesion, this field is growing and should provide new insights into the structural changes following stroke and how they may mediate restoration of function.

\section{Discussion \& recommendations}

This review highlights evidence of the value of looking beyond the focal lesion to better understand recovery after stroke; in particular, the role of interconnected networks. Advances in neuroimaging techniques are making this a reality. A summary of techniques that may be used to obtain this information, as discussed in the review, are outlined in Table 2 . Figure 3 provides an example of data that may be obtained to investigate functional and structural changes in brain networks before and after specific somatosensory rehabilitation $[140,141]$.

Models provide a synthesized, evidence-based framework for the translation of science to practice. On the basis of the literature reviewed, it is recommended that models of post-stroke recovery should be network-based and account for both functional and structural changes in the brain. These models can be used to: characterize the deficit and recovery; predict ability to benefit from therapy; and guide selection of individualized rehabilitation [142]. For example, a functional-anatomical model of post-stroke neglect has been described that highlights the contribution of neuronal dysfunction at remote sites on the nature of the deficit as well as the response to therapy [51]. Stinear et al. describe an algorithm to predict an individual's potential for upper limb recovery that includes an early clinical score, transcranial magnetic stimulation to assess functional integrity of CST and diffusion-weighted MRI to assess structural integrity of posterior limbs of the internal capsules [143]. A network model can also be used to identify how different therapies may help to re-establish interhemispheric connections [55] and/or facilitate reorganization via other networks such as attention networks. It may be used to generate hypotheses related to brain regions important in the recovery process and in rehabilitation.

Neuroimaging outcomes have the potential to be used as biomarkers and predictors of future outcome [144]. To date, it is unknown what 'baseline' level of structure and function is important to inform the recovery process. Neuroimaging techniques discussed in this review may help to address this question. The advanced imaging modalities reviewed above may also be used to help stratify patients and act as surrogate end points of recovery in clinical trials [145]. Importantly they have potential to provide novel insight in to neurobiological mechanisms underlying recovery.

Rehabilitation requires access to residual networks during the dynamic process of recovery; thus, we need to appreciate the capacity for these networks to change and interact. Resting-state FC analysis is well suited to the study of how 
Table 2. Summary of approaches that may be used to investigate morphological and functional changes in the brain associated with post-stroke recovery.

\begin{tabular}{|c|c|c|c|}
\hline Purpose & Acquisition & Analysis (information provided) & Potential applications \\
\hline Lesion characterization & FLAIR - 2D or 3D & $\begin{array}{l}\text { Identification and quantification of } \\
\text { volume and topography of the infarct }\end{array}$ & $\begin{array}{l}\text { Location of lesion - important relative to } \\
\text { putative function of known regions }\end{array}$ \\
\hline Penumbra & $\begin{array}{l}\text { Diffusion/perfusion MRI } \\
\text { or perfusion CT }\end{array}$ & $\begin{array}{l}\text { Identification and quantification of the } \\
\text { volume and topography of the } \\
\text { penumbra }\end{array}$ & $\begin{array}{l}\text { Identification of potentially salvageable } \\
\text { tissue for interventions such as tPA } \\
\text { Investigation of relationship with recovery }\end{array}$ \\
\hline $\begin{array}{l}\text { Task-related brain } \\
\text { activation }\end{array}$ & EPI BOLD contrast & $\begin{array}{l}\text { Visualization of brain activation } \\
\text { associated with an experimentally } \\
\text { controlled task }\end{array}$ & $\begin{array}{l}\text { Identification of regions in the brain that } \\
\text { are activated in response to a particular } \\
\text { task/stimulus }\end{array}$ \\
\hline WM tracts - 'integrity' & $\begin{array}{l}\text { DTI - fractional } \\
\text { anisotropy }\end{array}$ & Fiber tract structural characteristics & $\begin{array}{l}\text { Integrity of WM tracts for access in } \\
\text { training } \\
\text { Could be part of algorithm }\end{array}$ \\
\hline $\begin{array}{l}\text { WM fiber estimation } \\
\text { - tractography }\end{array}$ & $\begin{array}{l}\text { DTI with large number of } \\
\text { diffusion-weighted } \\
\text { directions (e.g., 60) }\end{array}$ & $\begin{array}{l}\text { WM tract estimation: } \\
\text { - Probability of connectivity of WM } \\
\text { tracts between two regions } \\
\text { - 'Whole-brain' and 'targeted' tracking } \\
\text { Apparent fiber density maps }\end{array}$ & $\begin{array}{l}\text { Structural connectivity of WM tracts } \\
\text { Visualize WM pathways supporting } \\
\text { eloquent functions in vivo } \\
\text { Quantify structural connections in vivo }\end{array}$ \\
\hline Functional connectivity & $\begin{array}{l}\text { fMRI - EPI } \\
\text { No particular task }\end{array}$ & $\begin{array}{l}\text { Intrinsic functional connectivity: } \\
\text { - Quantify strength and location of } \\
\text { coherence of spontaneous activity in } \\
\text { brain networks } \\
\text { - Quantify topology and properties of a } \\
\text { network }\end{array}$ & $\begin{array}{l}\text { Identify impact of stroke on remote } \\
\text { networks } \\
\text { Identify viable brain networks with } \\
\text { capacity for plasticity }\end{array}$ \\
\hline $\begin{array}{l}\text { Morphology of cortical } \\
\text { areas/gray matter }\end{array}$ & $\begin{array}{l}\text { T1 } 3 D \text { MPRAGE }- \text { high } \\
\text { resolution, isotonic } \\
(\text { e.g., } 1 \times 1 \times 1)\end{array}$ & $\begin{array}{l}\text { Quantify thickness and volume of } \\
\text { cortical and deep brain structures } \\
\text { Automated 3D volume analysis }\end{array}$ & $\begin{array}{l}\text { Identify atrophy over time } \\
\text { Quantify volume/cortical thickness of } \\
\text { putative brain areas } \\
\text { Identify change over time with recovery } \\
\text { and following intervention }\end{array}$ \\
\hline
\end{tabular}

multiple distributed networks are disrupted by and reorganize after stroke [10]. This information can be complemented by knowledge of structural connectivity ('the hardware'). Knowledge of disorders of network connectivity may also lead to development of novel interventions aimed at re-establishing those connections.

Neurobiological outcomes associated with current and future therapies may be probed using a number of the techniques reviewed (Table 2). For example, perfusion imaging provides important information about the hemodynamics in terms of affected area and degree of perfusion abnormality. Chronic carotid artery occlusion has been shown to go ahead with minor changes of the perfusion dynamics as assessed with maps of time to peak or mean transit time while the cerebral blood volume is not decreased and there are no or minor apparent diffusion coefficient changes [146,147]. Conversely, severe decreases of cerebral blood volume in acute stroke predict secondary hemorrhage after systemic thrombolysis [148]. Furthermore, perfusion MRI in combination with DWI may be used to assess the extent of penumbra pretreatment and to determine how much of the penumbra is salvaged by restoration of perfusion following reperfusion treatment. Advanced imaging, including task-related activation and FC, at later time points may be used to assess the extent to which reperfused areas are associated with longer-term recovery of specific functions and overall recovery.

Advanced imaging techniques may also be used as surrogate outcomes for rehabilitation therapies. Given the important role of brain networks in recovery, it is recommended that investigation of neuroanatomical outcomes before and after rehabilitation should include patterns of FC related to the specific function of interest, as well as networks that support rehabilitation and learning, such as attention networks. This will help to establish networks that have been accessed through therapy, as well as potential interconnections between networks. Knowledge of the structural characteristics and 


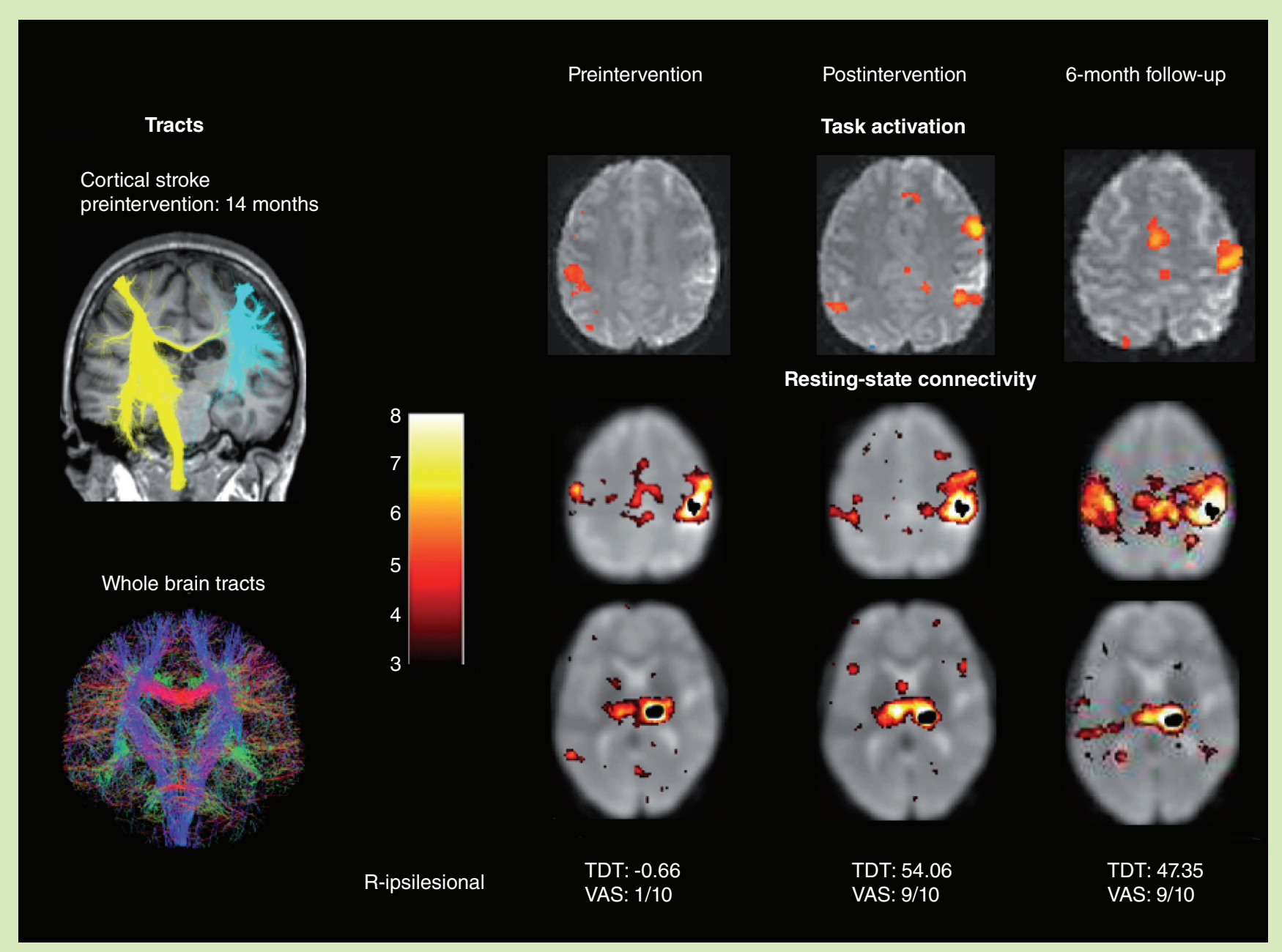

Figure 3. Example of MRI data to assess functional and structural changes in brain regions and networks before and after a 6-week period of sensory rehabilitation and at follow-up (6 months following the preintervention time) in a stroke survivor with somatosensory loss due to lesion of the primary somatosensory cortex. Touch discrimination, measured using the TDT was severely impaired (below chance TDT score and 1 out of 10 on VAS) prior to intervention and slightly below normal performance (TDT criterion = 66.1 area under the curve score) following intervention and at follow-up. Probability fiber tracts (top left), acquired using diffusion-weighted imaging tractography, show a relative lack of interhemispheric somatosensory cortex connectivity and reduced connectivity to ipsilesional thalamus when seeded from ipsilesional somatosensory cortex adjacent to the lesion. Task activation maps, obtained during a controlled touch task to the affected hand, show return to perilesional regions postintervention and at follow-up (top row). A relative increase in interhemispheric somatosensory cortex connectivity is observed at follow-up (middle row). TDT: Tactile Discrimination Test; VAS: Visual analog scale.

Adapted with permission from [141].

connectivity of white matter fibers within and beyond the lesion prior to intervention, using biologically reliable tractography, would also provide insight into brain networks important in recovery, help predict ability to benefit from rehabilitation, and provide information to guide individual targeting of therapy.

\section{Conclusion}

Insights into post-stroke recovery using advanced neuroimaging methods are changing the way we view recovery after stroke. The brain operates as a functional unit and is dynamic. This highlights the need to look beyond the focal lesion to better appreciate its impact on functional and structural brain networks, as well as identify the residual brain networks with capacity for plasticity. MRI offers advanced techniques to assess salvageable brain tissue, functional task-related activity, functional and structural connectivity of brain networks and morphological changes in the brain over time. These have been reviewed in the context of post-stroke recovery.

Advances have been made in understanding the relationship between patterns of brain activation and impaired function after stroke. Evidence is expanding in relation to different functions, such as movement, sensation and 
language, as well as in relation to different times post-stroke and under different intervention conditions. Key advances have been made in the role of brain networks in recovery $[10,106]$. A focal stroke lesion affects not only the local region but also the network to which it belongs. This may help to explain the variability in deficits across patients, as well as variability in response to therapy. Converging evidence from studies of FC highlight the role of interhemispheric connectivity and its relationship to impairment and recovery. In comparison, studies of effective connectivity highlight intrahemispheric connectivity within the network of interest. Taken together, these findings provide increasing evidence that impairment after stroke, such as motor deficits or neglect, results from changes in the overall network rather than from removal of a single component. It also implies the importance of adopting a network-based approach to guide the selection and development of interventions. Diffusion-based tractography is recommended to characterize fiber tracts and aid identification of spared structural connections within and between networks. Network-based models of recovery need to be systematically developed [142].

\section{Future perspective Characterize impact of lesion}

Traditionally the focus of post-stroke neuroimaging has been to define the location of the lesion relative to local anatomy. However, there is growing evidence that this does not adequately capture the nature of the deficit in relation to the information processing deficit or in relation to recovery. In particular, the need to look beyond the lesion to remote locations and functional networks is highlighted. It is envisaged that in the next 5-10 years information provided to the treating physician and rehabilitation therapist will include the extent to which the lesion interrupts known functional brain regions (e.g., such as motor or language), putative resting-state networks (both intra- and inter-hemispheric connections) and specific white matter tracts (such as CST) and their connections. One way this may be achieved is by overlaying the lesion on templates of important functional and structural networks to determine the extent of interruption. Over the next 5 years researchers will need to develop valid and robust templates in age-matched healthy controls for all of the major functional deficits. For example, templates of the functional motor network may be defined in vivo from a meta-analysis of existing task-related activation and/or connectivity data using activation likelihood estimation. As this evidence expands the potential exists to define probabilistic output of the likely functional deficit. Alternatively novel information of the extent of task-related activation, FC and structural connectivity may be acquired directly for an individual using techniques, such as intrinsic FC and diffusion-weighted tractography.

\section{Prediction}

How useful is neuroimaging in predicting outcomes in stroke rehabilitation [72]? In order to be useful for rehabilitation, neuroimaging needs to provide prognostic information for individual patients relatively early after stroke. Development of an algorithm for motor recovery that includes clinical, neurophysiological and imaging data have demonstrated the value of such an approach [143]. Advances in our understanding of the relationship between the clinical deficit, interruption to functional and structural connections, and outcome are necessary to inform prediction models. This field requires further systematic investigation over the next 5-10 years. Longitudinal studies with large sample size that span the acute to chronic phases and employ advanced neuroimaging modalities are needed to advance our knowledge of how the structural and FC of the brain evolves after stroke and is related to deficits and clinical outcomes. It is anticipated that methods that include combined functional and structural data relative to networks will be of value. In addition, the potential exists to test and refine algorithms that have been recently proposed. In this context, it is important that the individual patient is not burdened in the collection of unnecessary information. Given the 'open window of recovery' afforded by neural plasticity, the potential also exists at later stages in the recovery journey to assess an individual's capacity to benefit from a burst of a specific intervention. For example, this knowledge may be used to predict if there is sufficient residual capacity in the ipsilesional neural network to support a unilateral approach to motor recovery [149].

\section{Inform rehabilitation \& personalized medicine}

It is recommended that the rehabilitation clinician should be informed of interruption to specific functional networks targeted in therapy, for example, the motor system; relative integrity of putative networks of factors known to impact therapy, such as mood/depression; and residual 
strengths of networks that are important for learning, such as attention and memory. Lesion data could be overlaid on tract and functional data, as described above. Spared connectivity between key nodes in the network can then be identified. This focus on connectivity permits investigation of functional integration and interaction between distant brain areas. A resting-state $\mathrm{FC}$ acquisition of approximately 7 min could be obtained to inform intra- and inter-hemispheric connectivity of the above networks in the individual. This information should then be interpreted in the context of evidencebased models of recovery and rehabilitation. Over the next 5-10 years functional-anatomical models of recovery need to be systematically developed for the major functions commonly impaired after stroke. This information can then be used to guide the selection of therapies that most optimally target the viable brain networks

\section{Executive summary}

\section{Lesion characterization \& the penumbra}

- Early structural changes can be observed with diffusion-weighted MRI. This is followed by dynamic lesion evolution.

- Reperfusion and salvage of the ischemic penumbra are important in early recovery. The penumbra may be defined using techniques such as MRI perfusion/diffusion mismatch and may be impacted by interventions such as thrombolysis.

\section{Functional task-related activation \& recovery}

- Meta-analyses and systematic review of motor recovery studies indicate increased activation in contralesional primary motor cortex, bilateral premotor cortex and supplementary motor area relative to healthy controls.

- Better motor performance is associated with activation of ipsilesional primary motor cortex and the original motor network rather than contralesional activation.

- Similar changes in activation are observed following specific motor interventions.

\section{Brain networks \& impact on recovery}

- White matter fiber connectivity and integrity:

- Diffusion-based tractography may be used to quantify the structural integrity of white matter fiber tracts and to investigate white matter fiber connectivity. This has relevance for prediction of outcome and identification of spared structural connections within and between networks.

- It is critical that advanced imaging protocols and valid analysis methods are used in the characterization of fiber tracts in order to generate biologically reliable and valid tractography information.

- Functional connectivity of brain regions and networks:

- Functional brain networks may be investigated using resting-state (intrinsic) functional connectivity and/or effective connectivity of task-related activations. Methods have been developed to reflect local connectivity between regions of interest or properties and efficiencies of whole network connectivity.

- The focal lesion impacts the function of remote sites. These remote effects appear to remain within the bounds of the existing network connections.

- Alterations in the strength of intrinsic functional connections between hemispheres and between key regions within functional networks impacted by the lesion (e.g., motor network) have been described and related to behavioral deficits and their recovery after stroke. The role of interhemipsheric connections in recovery is highlighted.

- Changes in network efficiencies have also been identified over time, although the significance of these requires systematic investigation.

- Findings from effective connectivity analysis highlight changes in intrahemispheric interactions.

- Changes in intrinsic functional connectivity have been investigated in relation to a few interventions.

\section{Morphological changes over time}

- Voxel-based and surface-based methods have been employed to characterize changes in brain morphology over time and in association with recovery. Caution is required in interpretation given the impact of the lesion and related edema among others on categorization of brain structures.

- There are suggestions of changes in brain morphology associated with specific interventions; however, these require replication with rigorous methods of analysis.

\section{Conclusion}

- Advanced neuroimaging findings are changing the way we view recovery after stroke.

- We need to look beyond the lesion to changes in remote locations and brain networks.

- Increasing evidence supports the association between post-stroke recovery and changes in task-related activation, functional connectivity, structural integrity and connectivity, and cortical thickness.

- Converging evidence from studies of functional connectivity highlight the role of interhemispheric connectivity, while studies of effective connectivity highlight intrahemispheric connectivity within the network of interest.

- A multimodal approach to probe changes in brain networks is advocated. 
with capacity for plasticity. This will serve to inform a more personalized approach to medicine during the recovery journey [7].

\section{Technical \& analysis developments}

Given interactions between structural and functional changes in brain networks and the dynamic process of recovery, it will be important to adopt multimodal approaches to investigation and analysis. Taking the strengths from each, the potential exists to combine information to inform not only the nature of the deficit, but also the mechanisms that may underlie it. For example, task-related and resting-state data may be used to test hypotheses related to directional changes between regions in a network and across networks. Methods to investigate relationships between structural and functional networks need to be developed further. A recent example is the functional tract-weighted imaging [150]. Development of more sophisticated biologically interpretable models [151], methods of connectivity and multimodal analyses [152], and resolution of data acquired, such as accelerated fMRI with the ability to achieve subsecond whole brain imaging will all serve to take neuroimaging foundations of post-stroke recovery to the next level [153]. MRI has the strengths that is it noninvasive, has whole-brain coverage and good spatial resolution, but is currently limited in temporal resolution. Techniques such as magnetoencephalography and electroencephalography may be integrated with MRI to inform this dimension of change. Techniques such as transcranial magnetic stimulation may be used to experimentally test hypotheses.

\section{References}

Papers of special note have been highlighted as:

- of interest

-. of considerable interest

1. Mathers C, Fat DM, Boerma JT et al. Global Burden of Disease. 2004 Update. WHO, Geneva, Switzerland (2008).

2. Writing Group Members, Lloyd-Jones D, Adams RJ; American Heart Association. Heart disease and stroke statistics - 2010 update. Circulation 121(7), e46-e215 (2010).

3. Roger VL, Lloyd-Jones DM, Adams RJ et al.; American Heart Association Statistics Committee and Stroke Statistics Subcommittee. Heart disease and stroke statistics - 2011 update. Circulation 123(4), e18-e209 (2011).

4. Levin MF, Kleim JA, Wolf SL. What do motor 'recovery' and 'compensation' mean in patients following stroke? Neurorehabil. Neural. Repair 23(4), 313-319 (2009).

5. Kolb B, Teskey GC, Gibb R. Factors influencing cerebral plasticity in the normal and injured brain. Front Hum. Neurosci. 4, 204 (2010).

6. Carey LM. Stroke Rehabilitation: Insights from Neuroscience and Imaging. Oxford University Press, NY, USA (2012).

-. Provides a critical summary of insights from neuroscience and imaging in support of restorative approaches to stroke rehabilitation from a multidisciplinary group of experts in the field.

7. van Vliet P, Carey LM, Nilsson M. Targeting stroke treatment to the individual. Int. J. Stroke 7(6), 480-481 (2012).

8. Carey LM. Neuroplasticity and learning lead a new era in stroke rehabilitation. Int. J. Ther. Rehabil. 14(6), 200-201 (2007).
9. Carter AR, Astafiev SV, Lang CE et al. Resting interhemispheric functional magnetic resonance imaging connectivity predicts performance after stroke. Ann. Neurol. 67(3), 365-375 (2010).

- Investigates change in functional connectivity in attention and somatomotor networks, highlighting the behavioral significance of interhemispheric changes in both domains.

10. Carter AR, Shulman GL, Corbetta M. Why use a connectivity-based approach to study stroke and recovery of function? Neuroimage 62(4), 2271-2280 (2012).

11. Mohajerani MH, Aminoltejari K, Murphy TH. Targeted mini-strokes produce changes in interhemispheric sensory signal processing that are indicative of disinhibition within minutes. Proc. Natl Acad. Sci. USA 108(22), E183-E191 (2011).

12. Grefkes C, Fink GR. Reorganization of cerebral networks after stroke. New insights from neuroimaging with connectivity approaches. Brain 134(5), 1264-1276 (2011).

13. Kreisel SH, Hennerici MG, Bazner H. Pathophysiology of stroke rehabilitation. The natural course of clinical recovery, usedependent plasticity and rehabilitative outcome. Cerebrovasc. Dis. 23(4), 243-255 (2007).

14. Parsons MW, Christensen S, McElduff P et al. Pretreatment diffusion-and perfusion-MR lesion volumes have a crucial influence on clinical response to stroke thrombolysis. J. Cereb. Blood Flow Metab. 30(6), 1214-1225 (2010).

15. Seitz RJ, Oberstrass H, Ringelstein A, Wittsack HJ, Siebler M. Failed recovery from thrombolysis is predicted by the initial diffusion weighted imaging lesion. Cerebrovasc. Dis. 31(6), 580-587 (2011).
16. Campbell BC, Christensen S, Levi CR et al. Comparison of computed tomography perfusion and magnetic resonance imaging perfusion-diffusion mismatch in ischemic stroke. Stroke 43(10), 2648-2653 (2012).

- Important evidence from pooled data of large trials on MRI in acute stroke subjected to systemic thrombolysis.

17. Wheeler HM, Mlynash M, Inoue M et al. Early diffusion-weighted imaging and perfusion-weighted imaging lesion volumes forecast final infarct size in DEFUSE 2. Stroke 44(3), 681-685 (2013).

18. Kraemer M, Schormann T, Hagemann G, Bi Q, Witte O, Seitz R. Delayed shrinkage of the brain after ischemic stroke. Preliminary observations with voxel-guided morphometry. J. Neuroimaging 14, 265-272 (2004).

19. Talairach J, Tournoux P. Co-Planar Stereotaxic Atlas of the Human Brain. Thieme, NY, USA (1988).

20. Zhu LL, Lindenberg R, Alexander MP, Schlaug G. Lesion load of the corticospinal tract predicts motor impairment in chronic stroke. Stroke 41(5), 910-915 (2010).

21. Olsson E, Klasson N, Berge J et al. White matter lesion assessment in patients with cognitive impairment and healthy controls. Reliability comparisons between visual rating, a manual, and an automatic volumetrical MRI method - the Gothenburg MCI Study. J. Aging Res. 2013, 198471 (2013).

22. Seitz RJ, Donnan GA. Role of neuroimaging in promoting long term recovery from ischemic stroke. J. Magn. Reson. Imaging 32(4), 756-772 (2010).

23. Liepert J, Haevernick K, Weiller C, Barzel A. The surround inhibition determines 
therapy-induced cortical reorganization. Neuroimage 32(3), 1216-1220 (2006).

24. Abela E, Missimer J, Wiest R et al. Lesions to primary sensory and posterior parietal cortices impair recovery from hand paresis after stroke. PloS ONE 7(2), e31275 (2012).

25. Lindenberg R, Renga V, Zhu LL, Betzler F, Alsop D, Schlaug G. Structural integrity of corticospinal motor fibers predicts motor impairment in chronic stroke. Neurology 74(4), 280-287 (2010).

26. Chechlacz M, Rotshtein P, Humphreys GW. Neuroanatomical dissections of unilateral visual neglect symptoms. ALE meta-analysis of lesion-symptom mapping. Front. Hum. Neurosci. 6(230), 1-20 (2012).

27. Alstadhaug KB, Sjulstad A. Isolated hand paresis: a case series. Cerebrovasc. Dis. Extra 3(1), 65-73 (2013).

28. Bivard A, Levi C, Spratt N, Parsons M. Perfusion CT in acute stroke. A comprehensive analysis of infarct and penumbra. Radiology 267(2), 543-550 (2013).

- Large-scale systematic comparison of the accuracy of all commonly used perfusion computed tomography data postprocessing methods in the definition of infarct core and penumbra in acute stroke.

29. Miteff F, Levi CR, Bateman GA, Spratt N, McElduff P, Parsons MW. The independent predictive utility of computed tomography angiographic collateral status in acute ischaemic stroke. Brain 132(8), 2231-2238 (2009).

30. Davis SM, Donnan GA, Parsons MW et al. Effects of alteplase beyond 3 hours after stroke in the Echoplanar Imaging Thrombolytic Evaluation Trial (EPITHET): a placebo-controlled randomised trial. Lancet Neurol. 7(4), 299-309 (2008).

31. Kosior RK, Lauzon ML, Steffenhagen N, Kosior JC, Demchuk A, Frayne R. Atlas-based topographical scoring for magnetic resonance imaging of acute stroke. Stroke 41(3), 455-460 (2010).

32. Parsons M, Spratt N, Bivard A et al. A randomized trial of tenecteplase versus alteplase for acute ischemic stroke. $N$. Engl. J. Med. 366(12), 1099-1107 (2012).

33. Petersen SE, Dubis JW. The mixed block/event-related design. Neuroimage 62(2), 1177-1184 (2012).

34. Logothetis N, Pauls J, Augath M, Trinath T, Oeltermann A. Neurophysiological investigation of the basis of the fMRI signal. Nature 412, 150-157 (2001).

35. Stephan KE, Roebroeck A. A short history of causal modeling of fMRI data. Neuroimage 62(2), 856-863 (2012).
36. Eickhoff SB, Stephan KE, Mohlberg $\mathrm{H}$ et al. A new SPM toolbox for combining probabilistic cytoarchitectonic maps and functional imaging data. Neuroimage 25(4), 1325-1335 (2005).

37. Mazzetto-Betti KC, Leoni RF, Pontes-Neto OM et al. The stability of the blood oxygenation level-dependent functional MRI response to motor tasks is altered in patients with chronic ischemic stroke. Stroke 41(9), 1921-1926 (2010).

38. Carey LM, Abbott DF, Egan GF et al. Evolution of brain activation with good and poor motor recovery after stroke. Neurorehabil. Neural. Repair 20(1), 24-41 (2006).

39. Schaechter JD, Perdue KL. Enhanced cortical activation in the contralesional hemisphere of chronic stroke patients in response to motor skill challenge. Cereb. Cortex 18(3), 638-647 (2008).

40. Sharma N, Baron JC, Rowe JB. Motor imagery after stroke. Relating outcome to motor network connectivity. Ann. Neurol. 66(5), 604-616 (2009).

41. Buma FE, Lindeman E, Ramsey NF, Kwakkel G. Review: functional neuroimaging studies of early upper limb recovery after stroke: a systematic review of the literature. Neurorehabil. Neural. Repair 24(7), 589-608 (2010).

42. Carey LM, Abbott DF, Egan GF, Bernhardt J, Donnan GA. Motor impairment and recovery in the upper limb after stroke. Behavioral and neuroanatomical correlates. Stroke 36, 625-629 (2005).

43. Cramer SC, Shah R, Juranek J, Crafton KR, Le V. Activity in the peri-infarct rim in relation to recovery from stroke. Stroke 37, 111-115 (2006).

44. Grefkes C, Eickhoff SB, Nowak DA, Dafotakis M, Fink GR. Dynamic intra- and interhemispheric interactions during unilateral and bilateral hand movements assessed with fMRI and DCM. Neuroimage 41(4), 1382-1394 (2008).

45. Hodics T, Cohen LG, Cramer SC. Functional imaging of intervention effects in stroke motor rehabilitation. Arch. Phys. Med. Rehabil. 87(Suppl. 2), S36-S42 (2006).

46. Richards LG, Stewart KC, Woodbury ML, Senesac C, Cauraugh JH. Movementdependent stroke recovery. A systematic review and meta-analysis of TMS and fMRI evidence. Neuropsychologia 46(1), 3-11 (2008).

47. Rehme AK, Fink GR, Von Cramon DY, Grefkes C. The role of the contralesional motor cortex for motor recovery in the early days after stroke assessed with longitudinal fMRI. Cereb. Cortex 21(4), 756-768 (2011).
48. Rehme AK, Eickhoff SB, Rottschy C, Fink GR, Grefkes C. Activation likelihood estimation meta-analysis of motor-related neural activity after stroke. Neuroimage 59(3), 2771-2782 (2012).

49. Hubbard I, Carey LM, Budd T, Parsons MW. Brain activation and upper limb recovery post stroke. A systematic literature review. Int. J. Stroke. 7 (Suppl. 1), 28 (2012).

50. Saur D, Lange R, Baumgaertner A, Al E. Dynamics of language reorganization after stroke. Brain 129(6), 1371-1384 (2006).

51. Corbetta M, Kincade M, Lewis C, Snyder A, Sapir A. Neural basis and recovery of spatial attention deficits in spatial neglect. Nat. Neurosci. 8, 1603-1610 (2005).

52. Rossini PM, Altamura C, Ferreri F et al. Neuroimaging experimental studies on brain plasticity in recovery from stroke. Eura Medicophys. 43, 241-254 (2007).

53. Carey LM, Abbott DF, Harvey MR, Puce A, Seitz RJ, Donnan GA. Relationship between touch impairment and brain activation after lesions of subcortical and cortical somatosensory regions. Neurorehabil. Neural. Repair 25(5), 443-457 (2011).

54. Luft AR, Waller S, Forrester L et al. Lesion location alters brain activation in chronically impaired stroke survivors. Neuroimage 21(3), 924-935 (2004).

55. Carey LM, Seitz R. Functional neuroimaging in stroke recovery and neurorehabilitation. conceptual issues and perspectives. Int. J. Stroke 2(4), 245-264 (2007).

56. Stinear CM, Barber PA, Coxon JP, Fleming MK, Byblow WD. Priming the motor system enhances the effects of upper limb therapy in chronic stroke. Brain 131(5), 1381-1390 (2008).

57. Johansen-Berg H, Dawes H, Guy C, Smith S, Wade DT, Matthews PM. Correlation between motor improvements and altered fMRI activity after rehabilitative therapy. Brain 125, 2731-2742 (2002).

58. Dong Y, Dobkin BH, Cen SY, Wu AD, Winstein CJ. Motor cortex activation during treatment may predict therapeutic gains in paretic hand function after stroke. Stroke 37, 1552-1555 (2006).

59. Lin KC, Chung HY, Wu CY et al. Constraint-induced therapy versus control intervention in patients with stroke. A functional magnetic resonance imaging study. Am. J. Phys. Med. Rehabil. 89(3), 177-185 (2010).

60. Carey JR, Kimberley TJ, Lewis SM et al. Analysis of $\mathrm{fMRI}$ and finger tracking training in subjects with chronic stroke. Brain 125, 773-788 (2002). 
61. Whitall J, Waller SM, Sorkin JD et al. Bilateral and unilateral arm training improve motor function through differing neuroplastic mechanisms. A single-blinded randomized controlled trial. Neurorehabil. Neural. Repair 25(2), 118-129 (2011).

62. Page SJ, Szaflarski JP, Eliassen JC, Pan H, Cramer SC. Cortical plasticity following motor skill learning during mental practice in stroke. Neurorehabil. Neural. Repair 23(4), 382-388 (2009).

63. Michielsen ME, Selles RW, van der Geest JN et al. Motor recovery and cortical reorganization after mirror therapy in chronic stroke patients. A Phase II randomized controlled trial. Neurorehabil. Neural. Repair 25(3), 223-233 (2011).

64. Basser PJ. Inferring microstructural features and the physiological state of tissues from diffusion-weighted images. NMR Biomed. 8(7), 333-344 (1995).

65. Tournier JD, Mori S, Leemans A. Diffusion tensor imaging and beyond. Magn. Reson. Med. 65(6), 1532-1556 (2011).

66. Konishi J, Yamada K, Kizu O et al. MR tractography for the evaluation of functional recovery from lenticulostriate infarcts. Neurology 64(1), 108-113 (2005).

67. Newton JM, Ward NS, Parker GJ et al. Non-invasive mapping of corticofugal fibres from multiple motor areas - relevance to stroke recovery. Brain 129(Pt 7), 1844-1858 (2006).

68. Kunimatsu A, Itoh D, Nakata Y et al. Utilization of diffusion tensor tractography in combination with spatial normalization to assess involvement of the corticospinal tract in capsular/pericapsular stroke. Feasibility and clinical implications. J. Magn. Reson. Imaging 26(6), 1399-1404 (2007).

69. Dawes H, Enzinger C, Johansen-Berg $\mathrm{H}$ et al. Walking performance and its recovery in chronic stroke in relation to extent of lesion overlap with the descending motor tract. Exp. Brain Res. 186(2), 325-333 (2008).

70. Nelles M, Gieseke J, Flacke S, Lachenmayer L, Schild H, Urbach H. Diffusion tensor pyramidal tractography in patients with anterior choroidal artery infarcts. Am. J. Neuroradiol. 29(3), 488-493 (2008).

71. Stinear CM, Barber PA, Smale PR, Coxon JP, Fleming MK, Byblow WD. Functional potential in chronic stroke patients depends on corticospinal tract integrity. Brain 130(1), 170-180 (2007).

72. Stinear CM, Ward NS. How useful is imaging in predicting outcomes in stroke rehabilitation? Int. J. Stroke 8(1), 33-37 (2013).

73. Schaechter JD, Fricker ZP, Perdue KL et al. Microstructural status of ipsilesional and contralesional corticospinal tract correlates with motor skill in chronic stroke patients. Hum. Brain Mapp. 30(11), 3461-3474 (2009).

74. Jang SH, Ahn SH, Ha JS, Lee SJ, Lee J, Ahn YH. Peri-infarct reorganization in a patient with corona radiata infarct. A combined study of functional MRI and diffusion tensor image tractography. Restor. Neurol. Neurosci. 24(2), 65-68 (2006).

75. Cho S-H, Kim SH, Choi BY et al. Motor outcome according to diffusion tensor tractography findings in the early stage of intracerebral hemorrhage. Neurosci. Lett. 421(2), 142-146 (2007).

76. Pannek K, Chalk JB, Finnigan S, Rose SE. Dynamic corticospinal white matter connectivity changes during stroke recovery. A diffusion tensor probabilistic tractography study. J. Magn. Reson. Imaging 29(3), 529-536 (2009).

77. Basser PJ, Mattiello J, Lebihan D. MR diffusion tensor spectroscopy and imaging. Biophys. J. 66(1), 259-267 (1994).

78. Jeurissen B, Leemans A, Tournier JD, Jones DK, Sijbers J. Investigating the prevalence of complex fiber configurations in white matter tissue with diffusion magnetic resonance imaging. Hum. Brain Mapp. doi:10.1002/hbm.22099 (2012) (Epub ahead of print)

79. Farquharson S, Tournier JD, Calamante F et al. White matter fiber tractography. Why we need to move beyond DTI. J. Neurosurg. 118(6), 1367-1377 (2013).

-. Provides the first extensive and systematic exploration of the practical limitations of diffusion tensor imaging-based tractography and investigates whether the higher-order tractography model, known as constrained spherical deconvolution, provides a reasonable solution to these problems within a clinically feasible timeframe.

80. Tournier JD, Calamante F, Connelly A. Robust determination of the fibre orientation distribution in diffusion MRI. non-negativity constrained super-resolved spherical deconvolution. Neuroimage 35(4), 1459-1472 (2007).

81. Fernandez-Miranda JC, Pathak S, Engh J et al. High-definition fiber tractography of the human brain: neuroanatomical validation and neurosurgical applications. Neurosurgery 71(2), 430-453 (2012).

82. Raffelt D, Tournier JD, Rose $S$ et al. Apparent fibre density. a novel measure for the analysis of diffusion-weighted magnetic resonance images. Neuroimage 59(4), 3976-3994 (2012).
83. Smith RE, Tournier JD, Calamante F, Connelly A. SIFT: spherical-deconvolution informed filtering of tractograms. Neuroimage 67, 298-312 (2013).

84. Tournier J, Calamante F, Connelly A. MRtrix. Diffusion tractography in crossing fiber regions. Int. J. Imag. Syst. Tech. 22(1), 53-66 (2012).

85. Van Essen DC, Ugurbil K, Auerbach E et al. The Human Connectome Project. A data acquisition perspective. Neuroimage 62(4), 2222-2231 (2012).

86. Shmuel A, Leopold DA. Neuronal correlates of spontaneous fluctuations in fMRI signals in monkey visual cortex. Implications for functional connectivity at rest. Hum. Brain Mapp. 29(7), 751-761 (2008).

87. Waites AB, Stanislavsky A, Abbott DF, Jackson GD. Effect of prior cognitive state on resting state networks measured with functional connectivity. Hum. Brain Mapp. 24, 59-68 (2005).

88. Deco G, Corbetta M. The dynamical balance of the brain at rest. Neuroscientist 17(1), 107-123 (2011).

89. Shehzad Z, Kelly AM, Reiss PT et al. The resting brain. Unconstrained yet reliable. Cereb. Cortex 19(10), 2209-2229 (2009).

90. Van Dijk KR, Hedden T, Venkataraman A, Evans KC, Lazar SW, Buckner RL. Intrinsic functional connectivity as a tool for human connectomics. theory, properties, and optimization. J. Neurophysiol. 103(1), 297-321 (2010).

91. Biswal BB, Mennes M, Zuo XN et al. Toward discovery science of human brain function. Proc. Natl Acad. Sci. USA 107(10), 4734-4739 (2010).

92. Bullmore E, Sporns O. Complex brain networks: graph theoretical analysis of structural and functional systems. Nat. Rev. Neurosci. 10(3), 186-198 (2009).

93. Fox MD, Snyder AZ, Vincent JL, Corbetta M, Van Essen DC, Raichle ME. The human brain is intrinsically organized into dynamic, anticorrelated functional networks. Proc. Natl Acad. Sci. USA 102(27), 9673-9678 (2005).

94. Wang J, Zuo X, He Y. Graph-based network analysis of resting-state functional MRI. Front. Syst. Neurosci. 4, 16 (2010).

95. Friston KJ, Dolan RJ. Computational and dynamic models in neuroimaging. Neuroimage 52(3), 752-765 (2010).

96. Mclntosh A, Gonzalez-Lima F. Structural equation modeling and its application to network analysis in functional brain imaging. Hum. Brain Mapp. 2(1-2), 2-22 (1994).

97. Cohen AL, Fair DA, Dosenbach NU et al. Defining functional areas in individual human brains using resting functional 
connectivity MRI. Neuroimage 41(1), 45-57 (2008).

98. Shirer WR, Ryali S, Rykhlevskaia E, Menon V, Greicius MD. Decoding subject-driven cognitive states with whole-brain connectivity patterns. Cereb. Cortex 22(1), 158-165 (2012).

99. Greicius MD, Krasnow B, Reiss AL, Menon V. Functional connectivity in the resting brain. A network analysis of the default mode hypothesis. Proc. Natl Acad. Sci. USA 100(1), 253-258 (2003).

100. Fox MD, Snyder AZ, Vincent JL, Raichle ME. Intrinsic fluctuations within cortical systems account for intertrial variability in human behavior. Neuron 56(1), 171-184 (2007).

101. Boly M, Balteau E, Schnakers C et al. Baseline brain activity fluctuations predict somatosensory perception in humans. Proc. Natl Acad. Sci. USA 104(29), 12187-12192 (2007).

102. Lewis CM, Baldassarre A, Committeri G, Romani GL, Corbetta M. Learning sculpts the spontaneous activity of the resting human brain. Proc. Natl Acad. Sci. USA 106(41), 17558-17563 (2009).

103. Vincent JL, Patel GH, Fox MD et al. Intrinsic functional architecture in the anaesthetized monkey brain. Nature 447(7140), 83-86 (2007).

104. Damoiseaux JS, Greicius MD. Greater than the sum of its parts. A review of studies combining structural connectivity and resting-state functional connectivity. Brain Struct. Funct. 213(6), 525-533 (2009).

105. Jiang L, Xu H, Yu C. Brain connectivity plasticity in the motor network after ischemic stroke. Neural Plast. 2013, 924192 (2013).

106. Rehme AK, Grefkes C. Cerebral network disorders after stroke. Evidence from imagingbased connectivity analyses of active and resting brain states in humans. J. Physiol. 591(1), 17-31 (2013).

- Recent review of functional connectivity outcomes after stroke based on functional resting-state correlations, effective connectivity models or graph theoretical analyses.

107. He BJ, Snyder AZ, Vincent JL, Epstein A, Shulman GL, Corbetta M. Breakdown of functional connectivity in frontoparietal networks underlies behavioral deficits in spatial neglect. Neuron 53(6), 905-918 (2007).

108. James GA, Lu ZL, VanMeter JW, Sathian K, $\mathrm{Hu} \mathrm{XP}$, Butler AJ. Changes in resting state effective connectivity in the motor network following rehabilitation of upper extremity poststroke paresis. Top. Stroke Rehabil. 16(4), 270-281 (2009).
109. Wang L, Yu CS, Chen $\mathrm{H}$ et al. Dynamic functional reorganization of the motor execution network after stroke. Brain 133(4), 1224-1238 (2010).

110. Lu J, Liu H, Zhang $\mathrm{M}$ et al. Focal pontine lesions provide evidence that intrinsic functional connectivity reflects polysynaptic anatomical pathways. J. Neurosci. 31(42), 15065-15071 (2011).

111. Park $\mathrm{CH}$, Chang WH, Ohn SH et al. Longitudinal changes of resting-state functional connectivity during motor recovery after stroke. Stroke 42, 1357-1362 (2011).

112. Carter AR, Patel KR, Astafiev SV et al. Upstream dysfunction of somatomotor functional connectivity after corticospinal damage in stroke. Neurorehabil. Neural. Repair 26(1), 7-19 (2012).

113. Yin D, Song F, Xu D et al. Patterns in cortical connectivity for determining outcomes in hand function after subcortical stroke. PLoS ONE 7(12), e52727 (2012).

114. Golestani AM, Tymchuk S, Demchuk A, Goodyear BG. Longitudinal evaluation of resting-state fMRI after acute stroke with hemiparesis. Neurorehabil. Neural. Repair 27(2), 153-163 (2013).

115. Nomura EM, Gratton C, Visser RM, Kayser A, Perez F, D’Esposito M. Double dissociation of two cognitive control networks in patients with focal brain lesions. Proc. Natl Acad. Sci. USA 107(26), 12017-12022 (2010).

- Provides evidence that the effect of anatomical damage extends beyond the lesioned area, but remains within the bounds of the existing network connections. Network independence is demonstrated through a double dissociation of lesion location.

116. Warren JE, Crinion JT, Lambon Ralph MA, Wise RJ. Anterior temporal lobe connectivity correlates with functional outcome after aphasic stroke. Brain 132(12), 3428-3442 (2009).

117. Bannister LC, Crewther SC, Gavrilescu M, Carey LM. Somatosensory networks in stroke survivors with somatosensory impairment: new insights from resting state functional connectivity. Presented at: European Stroke Conference 2010. Barcelona, Spain, 25-28 May 2010.

118. van Meer M. van der Marel K, Wang K et al. Recovery of sensorimotor function after experimental stroke correlates with restoration of resting-state interhemispheric functional connectivity. J. Neurosci. 30(11), 3964-3972 (2010).

119. Grefkes C, Nowak DA, Eickhoff SB et al. Cortical connectivity after subcortical stroke assessed with functional magnetic resonance imaging. Ann. Neurol. 63(2), 236-246 (2008).

120. Mintzopoulos D, Astrakas LG, Khanicheh A et al. Connectivity alterations assessed by combining fMRI and MR-compatible hand robots in chronic stroke. Neuroimage 47, T90-T97 (2009).

121. Grefkes C, Nowak DA, Wang LE, Dafotakis M, Eickhoff SB, Fink GR. Modulating cortical connectivity in stroke patients by rTMS assessed with fMRI and dynamic causal modeling. Neuroimage 50(1), 233-242 (2010).

122. Rehme AK, Eickhoff SB, Wang LE, Fink GR, Grefkes C. Dynamic causal modeling of cortical activity from the acute to the chronic stage after stroke. Neuroimage 55(3), 1147-1158 (2011).

123. Wang LE, Fink GR, Diekhoff S, Rehme AK, Eickhoff SB, Grefkes C. Noradrenergic enhancement improves motor network connectivity in stroke patients. Ann. Neurol. 69(2), 375-388 (2011).

124. Inman CS, James GA, Hamann S, Rajendra JK, Pagnoni G, Butler AJ. Altered restingstate effective connectivity of fronto-parietal motor control systems on the primary motor network following stroke. Neuroimage 59(1), 227-237 (2012).

125. Westlake KP, Hinkley LB, Bucci M et al. Resting state alpha-band functional connectivity and recovery after stroke. Exp. Neurol. 237(1), 160-169 (2012).

126. Sergi F, Krebs HI, Groissier B et al. Predicting efficacy of robot-aided rehabilitation in chronic stroke patients using an MRIcompatible robotic device. Conference proceedings. Conf. Proc. IEEE Eng. Med. Biol. Soc. 2011, 7470-7473 (2011).

127. Varkuti B, Guan C, Pan Y et al. Resting state changes in functional connectivity correlate with movement recovery for BCI and robotassisted upper-extremity training after stroke. Neurorehabil. Neural. Repair 27(1), 53-62 (2013).

128. Lusebrink F, Wollrab A, Speck O. Cortical thickness determination of the human brain using high resolution 3T and 7T MRI data. Neuroimage 70, 122-131 (2013).

129. Ashburner J, Friston KJ. Voxel-based morphometry - the methods. Neuroimage 11(6), 805-821 (2000).

130. Acosta O, Bourgeat P, Zuluaga MA, Fripp J, Salvado O, Ourselin S. Automated voxelbased $3 \mathrm{D}$ cortical thickness measurement in a combined Lagrangian-Eulerian PDE approach using partial volume maps. Med. Image Anal. 13(5), 730-743 (2009).

131. Stebbins GT, Nyenhuis DL, Wang C et al. Gray matter atrophy in patients with ischemic 
stroke with cognitive impairment. Stroke 39(3), 785-793 (2008).

132. Sachdev PS, Chen X, Joscelyne A, Wen W, Altendorf A, Brodaty H. Hippocampal size and dementia in stroke patients: the Sydney stroke study. J. Neurol. Sci. 260(1-2), 71-77 (2007).

133. Nitkunan A, Lanfranconi S, Charlton RA, Barrick TR, Markus HS. Brain atrophy and cerebral small vessel disease: a prospective follow-up study. Stroke 42(1), 133-138 (2011).

134. Brodtmann A, Pardoe H, Li Q, Lichter R, Ostergaard L, Cumming T. Changes in regional brain volume three months after stroke. J. Neurol. Sci. 322(1-2), 122-128 (2012).

135. Hickmott PW, Steen PA. Large-scale changes in dendritic structure during reorganization of adult somatosensory cortex. Nat. Neurosci. 8(2), 140-142 (2005).

136. Maguire EA, Gadian DG, Johnsrude IS et al. Navigation-related structural change in the hippocampi of taxi drivers. Proc. Natl Acad. Sci. USA 97(8), 4398-4403 (2000).

137. Draganski B, Gaser C, Busch V, Schuierer G, Bogdahn U, May A. Neuroplasticity: changes in grey matter induced by training. Nature 427(6972), 311-312 (2004).

138. Schaechter JD, Moore CI, Connell BD, Rosen BR, Dijkhuizen RM. Structural and functional plasticity in the somatosensory cortex of chronic stroke patients. Brain 129(10), 2722-2733 (2006).
- First evidence of colocalized functional (brain activation) and structural (cortical thickness) plasticity in the somatosensory cortex of chronic stroke patients.

139. Gauthier LV, Taub E, Perkins C, Ortmann M, Mark VW, Uswatte G. Remodeling the brain: plastic structural brain changes produced by different motor therapies after stroke. Stroke 39, 1520-1525 (2008).

140. Carey LM, Macdonnell R, Matyas T. SENSe: study of the effectiveness of neurorehabilitation on sensation.

A randomized controlled trial. Neurorehabil. Neural. Repair 25, 304-313 (2011).

141. Carey LM. Touch and body sensations. In: Stroke Rehabilitation: Insights from Neuroscience and Imaging. Carey LM (Ed.). Oxford University Press, NY, USA, 157-172 (2012).

142. Carey LM. Directions for stroke rehabilitation clinical practice and research. In: Stroke Rehabilitation: Insights from Neuroscience and Imaging. Carey LM (Ed.). Oxford University Press, NY, USA, 240-249 (2012).

143. Stinear CM, Barber PA, Petoe M, Anwar S, Byblow WD. The PREP algorithm predicts potential for upper limb recovery after stroke. Brain 135(Pt 8), 2527-2535 (2012).

144. Cramer SC, Sur M, Dobkin BH et al. Harnessing neuroplasticity for clinical applications. Brain 134, 1591-1609 (2011).

145. Cramer SC. Stratifying patients with stroke in trials that target brain repair. Stroke 41(10), S114-S116 (2010).
146. Surikova I, Meisel M, Siebler M, Wittsack HJ, Seitz RJ. Significance of the perfusion-diffusion mismatch in chronic cerebral ischemia. J. Magn. Reson. Imaging 24(4), 771-778 (2006).

147. Liebeskind DS. Imaging the future of stroke: II. Hemorrhage. Ann. Neurol. 68(5), 581-592 (2010).

148. Campbell BC, Christensen S, Parsons MW et al. Advanced imaging improves prediction of hemorrhage after stroke thrombolysis. Ann. Neurol. 73(4), 510-519 (2013).

149. Stinear C, Byblow W. Targeting viable brain networks to improve outcomes after stroke. In: Stroke Rehabilitation: Insights from Neuroscience and Neuroimaging. Carey LM (Ed.). Oxford University Press, NY, USA, 233-239 (2012).

150. Calamante F, Masterton RA, Tournier JD et al. Track-weighted functional connectivity (TW-FC): a tool for characterizing the structural-functional connections in the brain. Neuroimage 70, 199-210 (2013).

151. Brodersen KH, Schofield TM, Leff AP et al. Generative embedding for model-based classification of fMRI data. PLoS Comput. Biol. 7(6), e1002079 (2011).

152. Smith SM. The future of fMRI connectivity. Neuroimage 62(2), 1257-1266 (2012).

153. Feinberg DA, Moeller S, Smith SM et al. Multiplexed echo planar imaging for sub-second whole brain fMRI and fast diffusion imaging. PLOS ONE 5(12), e15710 (2010). 


\section{Medscape Beyond the lesion: neuroimaging foundations for post-stroke recovery}

To obtain credit, you should first read the journal article. After reading the article, you should be able to answer the following, related, multiple-choice questions. To complete the questions (with a minimum $70 \%$ passing score) and earn continuing medical education (CME) credit, please go to www.medscape.org/journal/fnl. Credit cannot be obtained for tests completed on paper, although you may use the worksheet below to keep a record of your answers. You must be a registered user on Medscape.org. If you are not registered on Medscape.org, please click on the New Users: Free Registration link on the left hand side of the website to register. Only one answer is correct for each question. Once you successfully answer all post-test questions you will be able to view and/or print your certificate. For questions regarding the content of this activity, contact the accredited provider,
CME@medscape.net. For technical assistance, contact CME@webmd.net. American Medical Association's Physician's Recognition Award (AMA PRA) credits are accepted in the US as evidence of participation in CME activities. For further information on this award, please refer to http://www.ama-assn.org/ama/pub/ category/2922.html. The AMA has determined that physicians not licensed in the US who participate in this CME activity are eligible for AMA PRA Category 1 Credits $^{\mathrm{TM}}$. Through agreements that the AMA has made with agencies in some countries, AMA PRA credit may be acceptable as evidence of participation in CME activities. If you are not licensed in the US, please complete the questions online, print the AMA PRA CME credit certificate and present it to your national medical association for review.

\section{Activity evaluation: where 1 is strongly disagree and 5 is strongly agree.}

The activity supported the learning objectives.

The material was organized clearly for learning to occur.

The content learned from this activity will impact my practice.

The activity was presented objectively and free of commercial bias.

1. Your patient is a 67-year-old male seen 2 hours after onset of symptoms from right middle cerebral artery stroke. Based on the review by Dr. Carey and colleagues, which of the following statements about the role of magnetic resonance imaging (MRI) in elucidating structural changes affecting poststroke recovery is most likely correct?
A Diffusion-weighted MRI does not visualize early structural changes
B Reperfusion and salvage of the ischemic penumbra do not affect recovery
C MRI perfusion/diffusion mismatch may help define the penumbra
D Interventions such as thrombolysis do not affect the penumbra

2. Which of the following statements about connectivity of brain regions and networks visualized by neuroimaging techniques in poststroke recovery is most likely correct?
A Diffusion-based tractography may help to quantify the structural integrity of white matter fiber tracts and to assess white matter fiber connectivity
B White matter fiber connectivity is not a predictor of outcome
C Interhemispheric connectivity does not affect impairment or recovery
D Motor deficits after stroke result exclusively from removal of a single brain component 
3. Which of the following statements about functional activation in poststroke recovery as determined by neuroimaging techniques would most likely be correct?

A Better motor performance is associated with activation of primary motor cortex (M1) on the opposite side, rather than the same side, as the lesion

B During motor recovery, there is decreased activation in bilateral ventral premotor cortex (PMC) and supplementary motor area (SMA) compared with healthy controls

C The focal lesion affects the function of remote sites, within the bounds of the existing network connections

D The significance of changes in network efficiencies over time is well understood 